\title{
Some effects of remembering on forgetting
}

\author{
WILLARD N. RUNQUIST \\ University of Alberta, Edmonton, Alberta, Canada
}

\begin{abstract}
Three experiments investigated the effect of testing for recall on forgetting. One experiment demonstrated that the effect of testing is restricted to the items tested. A second experiment showed that the difference between tested and untested items increased with longer retention intervals. Increasing the study time had no effect on the retention of tested items, but increased the recallability of untested items. A third experiment ruled out rehearsal during the retention interval as an explanation. It was concluded that the critical aspects of a test are the retrieval operations themselves. Suggestions are made as to how these operations attenuate forgetting.
\end{abstract}

Both common sense and countless experiments indicate that information in memory becomes less accessible when it is not activated or utilized for some time. This decrease in accessibility is commonly called "forgetting." It is also well established that retrieving information from memory renders that information more accessible upon some later occasion.

The research reported in this paper was concerned with testing effects on the course of forgetting over relatively long periods of time. The experiments were motivated primarily by certain conceptual and methodological problems involved in the study of forgetting, but the results have important implications for the understanding of testing effects in general.

The problem originates with the very definition of forgetting. Conceptually, the notion of forgetting implies that information that was once accessible can no longer be retrieved. Operationally, then, the measurement of forgetting requires two retrieval tests separated by a time interval. Working under the assumption that any event that is perceived is immediately available (e.g., Tulving, 1964), some researchers may not provide the initial test, but forgetting can only be demonstrated by determining the actual accessibility of that information at two different times. Normally, the first test is given as close to the end of input as is possible, but there is no logical necessity for this restriction, and forgetting may be validly measured between any two points in time.

Although the general operations required to measure forgetting are straightforward, the realization of concrete procedures presents somewhat of a problem. Two kinds of design are available. One procedure is to give both tests to the same person at two different times. This permits analyses of the fate of the individual items

This study was supported by Research Grant A4867 from the Natural Sciences and Fngineering Research Council of Canada. Louise Renncy and Linda Butler supervised the data collection. Send reprint requests to $W$. N. Runquist, Department of Psychology, University of Alberta, Edmonton, Alberta T6G 2F9, Canada. of information that were remembered on the first test, but confounds forgetting with testing effects. Whatever testing procedure is used to assess the initial accessibility of the information will result, in all likelihood, in changes in the memorial systems, changes that could affect the course of forgetting. Moreover, forgetting technically refers only to information initially remembered, so that only information that is easiest to store is available on the initial test. Actual measurements of memorability may be confounded by regression effects (Runquist, 1973).

The second design requires testing different persons on the two tests, thus avoiding the above problems, but at the expense of losing the history of individual items of information. In this case, forgetting must be defined as less total information recalled on the later test than on the earlier test.

The dilemma is essentially irresolvable, although it is possible to incorporate the features of both procedures into a single design as long as testing involves cuing for specific items of information. Immediately after input, all subjects are tested for some of the information acquired. After varying retention intervals, subjects may be tested on all information. Performance on the untested information on the second test should provide a measure of forgetting unbiased by testing effects. Performance on the previously tested information is subject to testing effects but still may be analyzed in detail to provide an indication of changes in the recallability of specific items of information.

The particular way in which the initial test affects memory is crucial to the interpretation of forgetting data. If, as some researchers have maintained, a test trial merely acts as further study (e.g., Thompson, Wenger, \& Bartling, 1978), then no particular interpretive problem exists. One may simply treat testing as producing a "stronger trace." On the other hand, other researchers have taken the position that the learning that occurs on a test involves mechanisms different from those in effect during study (e.g., Allen, Mahler, \& Estes, 1969). In this case, the processes involved in forgetting may not be comparable. Furthermore, it is possible that 
the mechanisms involved may depend upon the task demands of a particular situation (Martin, Fleming, \& Nally, 1978).

The purpose of these experiments was to explore certain facets of testing effects in a cued-recall paradigm. The basic procedure of the experiments was as described above. The subjects studied pairs of words. They were then given a cued-recall test for one-half of the words. After retention intervals of a few minutes, several days, or weeks, they were given a cued-recall test for all of the words on the list. The critical results in each experiment involved comparisons of recall of previously tested and untested items at various retention intervals.

\section{EXPERIMENT 1}

The purpose of the first experiment was to determine whether testing effects are restricted to the items tested or are transferred to items that have not been tested. The issue is important for two reasons. First, if testing effects are item specific, general practice in retrieving would seem to be ruled out as a factor (LaPorte \& Voss, 1975). Second, the purpose of including the untested items on the second test is to assess forgetting in the absence of testing effects. If the two sets of items interact, the results on untested items are not serving their purpose.

Also included in the experiment was a condition in which the initial test was a recognition test and the delayed test was a recall test. If testing effects result from the further study of items that are recalled, a recognition test might produce even greater testing effects, since the subject would have the opportunity to study items that might have been inaccessible on a recall test. On the other hand, if testing effects involve the acquisition of retrieval strategies of some kind, a recognition test might not produce as much facilitation as a recall test.

\footnotetext{
Method

Each subject studied and was tested on a list of 24 word pairs. Two lists were used, with half of the subjects in each condition studying each list. The lists were those used by Tulving and Thomson (1973) and consisted of weakly associated pairs of words.

In studies of long-term retention, it is important to control active rehearsal during the retention interval, especially since this rehearsal could interact with testing effects. This issue was addressed more directly in Experiment 3, but for the present it will be sufficient to simply describe the procedure used to provide this control. The subjects made appointments for two sessions separated by the appropriate retention interval. They were then told that the experiment involved taking a battery of tests, ostensibly to determine the correlations among a number of different tasks.

The first test was the memory test. Each of the 24 word pairs was presented visually to the subject for $3 \mathrm{sec}$. The target word appeared in uppercase and the cue word in lowercase. The instructions were the nonspecific associative-learning instructions used by Tulving and Thomson (1973). Immediately af ter study, the subject worked on a set of mathematical-series problems for $2 \mathrm{~min}$.
}

Four groups of subjects were then given a test for their memory of 12 of the word pairs. For two groups, the test was a cued-recall test. Twelve cues were listed on a piece of paper, and the subject tried to write in the correct target for each cue. For the remaining two groups, the test was a pair-recognition test. Each of 12 pairs from the studied list was listed next to a pair from the list not studied. The subject had to indicate which of the two pairs was the one that had been studied. This test was allotted $2 \mathrm{~min}$. Two other groups did not have a memory test. Instead, the subjects were told that no memory test would be given, and that the purpose of learning the word list was to see whether it would interfere with the performance of the other tests.

All subjects then worked on the Ravens Progressive Matrices test for $10 \mathrm{~min}$. This test presents geometric forms with a section missing. The subject is required to choose the missing section from a set of alternatives.

One of the groups that had had the recall test, one of the groups that had had the recognition test, and one of the groups that had had no memory test were then given a 4-min cued-recall test on all 24 items. Cues were arranged 12 to a page, with previously tested cues on one page and untested cues on the other. The subjects in the remaining three groups were dismissed and instructed to return 1 week later to complete the test battery. At that time, they were given the cued-recall test as described above.

Thus, the factors in the design were: (1) recall, recognition, or no initial test and (2) 10-min or 1-week retention interval, as measured from the termination of the initial test.

There are a number of other details that should be documented. Each list was arbitrarily divided into two sets of 12 items. On the initial test, each set was used for half of the subjects in each group. There were six different presentation orders for each list and four different test orders for each page. Presentation and test orders were not balanced, however. On the second test, half of the subjects had the tested items and half had untested items on the first page. On the recognition test, the target-lure pairs were the same for all subjects. The lures were paired with the same targets on both tests.

Word pairs were presented on cards. The subjects had decks of 24 cards and turned over each card upon an auditory signal. The subjects were 144 introductory psychology students, who participated as part of a laboratory requirement for the course The subjects were tested in groups of up to 6 students. Conditions were assigned according to a predetermined randorn arrangement. An additional 52 subjects were tested or partially tested, but their data were rejected. Most rejections resulted from failure to return for the second session. The rather high rate of rejection also resulted from the fact that an experimenter error could invalidate the data of six subjects at a time.

\section{Results}

The mean number of items recalled on the first test was 6.33 for the $10-\mathrm{min}$ group and 6.70 for the 1-week group. The mean number of items correctly recognized was 10.42 for the $10-\mathrm{min}$ group and 11.00 for the 1-week group.

The mean number of items recalled on the second test is presented in Table 1 . For the groups that did not have the initial test, the division of tested and untested items was made by yoking items with those in the recognition or recall groups. It would appear that the recall of untested items on the second test was not affected by the initial test at either the short or the long retention interval.

Since this conclusion involves tacit acceptance of the null hypothesis, it is more convincing to make specific 
Table 1

Mean Number of Items Recalled for Previously Tested and Previously Untested Items as a Function of Retention Interval and Conditions of Previous Testing

\begin{tabular}{|c|c|c|c|}
\hline \multirow{2}{*}{$\begin{array}{c}\text { Tested/ } \\
\text { Untested }\end{array}$} & \multicolumn{3}{|c|}{ Previous Test } \\
\hline & None & Recall & Recognition \\
\hline \multicolumn{4}{|c|}{ 10-min Retention Interval } \\
\hline $\begin{array}{l}\text { Tested } \\
\text { Untested }\end{array}$ & $\begin{array}{l}4.79 \\
4.50\end{array}$ & $\begin{array}{l}6.33 \\
4.29\end{array}$ & $\begin{array}{l}7.25 \\
3.75\end{array}$ \\
\hline \multicolumn{4}{|c|}{ 1-week Retention Interval } \\
\hline $\begin{array}{l}\text { Tested } \\
\text { Untested }\end{array}$ & $\begin{array}{r}.83 \\
1.33\end{array}$ & $\begin{array}{r}4.20 \\
.46\end{array}$ & $\begin{array}{r}1.96 \\
.82\end{array}$ \\
\hline
\end{tabular}

comparisons between critical groups than to conduct an omnibus analysis of variance. Thus, separate $t$ tests were used to compare recall on the second test for the control group with that for the group that had had recall on the initial test and with that for the group that had had recognition on the initial test. Separate comparisons were made for tested and untested items.

The groups that had had an initial recall test recalled more tested items than did the control group after $10 \mathrm{~min}[\mathrm{t}(40)=2.08]$ and after 1 week $[\mathrm{t}(46)=5.56]$. In other words, there was a substantial testing effect. For the untested items, however, neither difference was significant $[\mathrm{t}(46)=.66, \mathrm{p}=.51$, after $10 \mathrm{~min}$ and $\mathrm{t}(46)=.90, \mathrm{p}=.37$, after 1 week] .

Groups that had had an initial recognition task also showed testing effects, but the effects were significant only for the 10 -min retention interval $[\mathrm{t}(46)=3.08]$. For the 1-week retention interval, $t(46)=1.81, \mathrm{p}=.08$. For the untested items, again, neither difference was significant. For the $10-\mathrm{min}$ interval, $\mathrm{t}(46)=1.07, \mathrm{p}=.29$, and for the 1-week interval, $\mathrm{t}(46)=1.35, \mathrm{p}=.18$.

Recall after 1 week was very poor for untested items. Although this may indicate the need for caution in accepting the null hypothesis for the 1-week retention interval, no such reservation need apply to the short retention interval.

It also appears that the recognition and recall tests do produce different effects on later recall. After $10 \mathrm{~min}$, there was no difference in the mean number of tested items recalled between the group whose initial test had been recall and the group whose initial test had been recognition $[\mathrm{t}(46)=1.06, \mathrm{p}=.30]$. After 1 week, however, more tested items were recalled when the initial test had been a recall test $[t(46)=3.88]$. For untested items, the difference between these groups was not significant at either the $10-\mathrm{min}[\mathrm{t}(46)=.60$, $\mathrm{p}=.56]$ or the 1 -week $[\mathrm{t}(46)=.94, \mathrm{p}=.35]$ interval.

For the groups that had had the immediate test, it is possible to examine performance on particular items on the second test conditionalized on whether they were remembered or not remembered on the initial test. The results of this analysis are presented in Table 2 . The most striking feature of these data is the strong depen-
Table 2

Proportion of Items Recalled on Second Test as a

Function of Retention Interval and Conditions of Previous Testing for Items Correct and Items Incorrect on Previous Test

\begin{tabular}{|c|c|c|}
\hline \multirow{2}{*}{$\begin{array}{l}\text { Correct/ } \\
\text { Incorrect }\end{array}$} & \multicolumn{2}{|c|}{ Previous Test } \\
\hline & Recall & Recognition \\
\hline \multicolumn{3}{|c|}{ 10-min Retention Interval } \\
\hline $\begin{array}{l}\text { Correct } \\
\text { Incorrect }\end{array}$ & $\begin{array}{l}.92 \\
.06\end{array}$ & $\begin{array}{l}.66 \\
.21\end{array}$ \\
\hline \multicolumn{3}{|c|}{ 1-week Retention Interval } \\
\hline $\begin{array}{l}\text { Correct } \\
\text { Incorrect }\end{array}$ & $\begin{array}{l}.60 \\
.04\end{array}$ & $\begin{array}{l}.17 \\
.05\end{array}$ \\
\hline
\end{tabular}

dence of recall on recall. In order to be recalled on the second test, an item must almost always have been recalled on the initial test. Recall does not depend as strongly on recognition, with fewer recognized items being recalled. Also of some interest is the considerable proportion of nonrecognized items that were recalled after the 10-min interval.

\section{Discussion}

There was no evidence in these data that the initial test had any effect at all on the untested items. No claims are made for the generality of this result, however. It is likely that materials and/or procedures that encourage the storage of information in organized or structured fashion rather than as separate units might result in some interaction among items. In fact, Mandler and Rabinowitz (1981) have obtained considerable "spread of accessibility" in experiments involving recog. nition and free recall of categorizable lists acquired by a categorizing procedure. This caveat does not rescue theories proposing that general practice at retrieval is a major component of testing effects (LaPorte \& Voss, 1975). Indeed, for the present design to provide critical evidence on this issue, it is necessary that possible sources of item interaction be eliminated. There was no evidence to support the existence of a general practice effect in this experiment.

The recognition test did produce testing effects of reasonable magnitude, but these effects differed in a number of ways from those produced by the recall test. After $10 \mathrm{~min}$, the overall testing effects produced by the two tests were quantitatively indistinguishable, but after 1 week, the recognition test produced less facilitation than did the recall test. Moreover, even at the 10-min interval, there were differences in the conditional relations between second-test recall and performance on the initial test. These differences are not consistent with a theory that assumes that testing effects result from the extra study provided by the test (Thompson et al., 1978).

The most direct evidence is, of course, the poorer recall performance following recognition than following recall after a long retention interval, but before the 
extra-study hypothesis can be laid to rest, some paradoxical effects that might result from the recognition test should be considered.

In theory, the recognition test should provide a study advantage. If it is assumed that the subject rehearsed or otherwise processed items that were correctly retrieved (on either test), more items were accessible for further processing during the recognition test than during the recall test. This is true, incidentally, even though some of the correct recognitions were actually only guessed correct. (Corrected for guessing, the subjects still averaged $73 \%$ correct responses on the recognition test, compared with $53 \%$ correct on the recall test.) The implication of this analysis is that, given a constant amount of time for the test, a subject executing recognition might have distributed processing time among more items. Some of these items were undoubtedly items that would not have been recallable. Thus, in terms of a second recall test, the initial recall test could result in "strengthening" the accessibility of the items already recallable, whereas the recognition test could enable the subject to add some items to the pool of recallable items, but at the expense of not strengthening already recallable items quite as effectively. In the short term, the recognition test might provide an advantage, but in the long term, the individual items are more fragile and susceptible to forgetting.

The conditional-recall data are in part consonant with this interpretation. Regression effects notwithstanding, items that were correctly recalled on the initial test were more likely to be correctly recalled on the second test than were items that were correctly recognized. This was true irrespective of retention interval. Nevertheless, the data are not entirely convincing in this respect. First, there is the higher rate of recall of items that were not correctly recognized. It seems irrational that the subject would consistently choose one pair as being correct and then study the other pair, although some increment in recallability could result from the processing necessary to make the recognition decision. Second, the lower conditional recall of correctly recognized items could result from the fact that some of the correctly recognized items were only guessed correctly. It seems much more reasonable to assume that, all in all, the differences in conditional recall represent some independence in the retrieval operations involved on the two tests, and do not result from differences in rehearsal.

If subjects do rehearse items that they recall and recognize, then one might expect them to sometimes study errors. There are several consequences of this assumption. Consider first just the groups that had had recall on the initial test. On the second test, overt errors on tested items should be more frequent than overt errors on untested items. This is clearly untrue. After $10 \mathrm{~min}$, the mean number of overt errors was 2.7 on tested items and 2.4 on untested items. After 1 week, the means were 3.4 and 3.7 , respectively. It is also significant that error frequency in tested items did not differ from error frequencies obtained in the control group (2.8 after $10 \mathrm{~min}$ and 3.1 after 1 week). To some extent, subjects do tend to repeat the errors on the second test that had been made on the initial test ( $47 \%$ after $10 \mathrm{~min}$ and $51 \%$ after 1 week), but this is probably not of theoretical consequence. Not only is the percentage independent of retention interval, but also most of the errors ( $80 \%$ ) could be classified as free associates to the cue. Thus, there was little evidence of error rehearsal in the recall groups.

The situation on the recognition test is somewhat different in that the frequency of errors is the complement of correct choices. Since the frequency of errors on the initial test was low, error rehearsal should be minimal. Interestingly enough, these subjects did make fewer errors following recognition than following recall (1.3 after $10 \mathrm{~min}$ and 2.9 after 1 week), but, again, there was no difference between the results for the tested and those for the untested items. The latter items produced 2.0 errors after $10 \mathrm{~min}$ and 2.8 errors after 1 week. Furthermore, it may be noted that the number of intrusions of lures on the final recall test was small. Due to the vagaries of the Tulving-Thomson lists, some intrusions occur as "free" associates to the cues, but the frequency of these errors in the groups that had had the recognition test did not exceed that in the groups that had not seen the lures. For the $10 \mathrm{~min}$ group, the mean was .46 intrusions; for the 1-week group, the mean was .20 intrusions. For the two other groups combined, the means were .21 after $10 \mathrm{~min}$ and .75 after 1 week. There was little evidence of error rehearsal in the recognition groups as well.

In summary, the results of this experiment appear to be more consistent with the assumption that testing effects result from processes different from those occurring on study trials. A consideration of the nature of these processes will be postponed until the results of two other experiments have been presented. It should be noted, however, that the effects of a recognition test transferred to recall. Wenger, Thompson, and Bartling (1980) have shown the inverse, namely, transfer from recall to recognition. Whether the effects are symmetrical is indeterminate, since the differences between their study and the present one are immense.

\section{EXPERIMENT 2}

Wenger et al. (1980) suggested that facilitation produced by testing is greater at longer retention intervals. The results of Experiment 1 are at least descriptively in accord with this conclusion. The purpose of Experiment 2 was to examine the effect of prior testing and level of initial recall on the rate of forgetting. If testing effects reflect the same processes that are involved in study effects, both variables should have the same effect on the course of forgetting. 


\section{Method}

The word lists and basic procedure were the same as for the recall conditions of Experiment 1. This procedure was as follows: (1) Each subject studied 24 pairs, with each pair being presented either once or three times. (2) Mathematical series problems were given for $2 \mathrm{~min}$. (3) All subjects received a 2-min written cued-recall test for 12 items. (4) The Ravens Progressive Matrices test was given for $10 \mathrm{~min}$. (5) There were six different retention intervals, ranging from immediate to 3 weeks. (6) All subjects spent $5 \mathrm{~min}$ on a cartoon-rating task. (7) The final test was a cued-recall test for all 24 items.

The six retention intervals were labeled 0,1 , and $6 \mathrm{~h}$ and 2, 7, and 21 days. Actually, they were determined as follows. For the 0 interval, the cartoon rating and the final test followed the Ravens test immediately. The interval was thus approximately $20 \mathrm{~min}$ from the end of acquisition. All other subjects left the laboratory immediately after the Ravens test and returned after an appropriate interval. There was some variability in actual retention interval due to variability in the subjects' actual time of arrival for both the initial session and the retention test. The point of reference for the nominal retention intervals listed above was the time of the initial appointment. Thus, the 1-h subjects returned on the next hour, the 6-h subjects 6 h later, etc.

All balancing procedures with regards to tests and test orders were the same as for Experiment 1.

The subjects were 288 introductory psychology students who were assigned to conditions and tested as in Fxperiment 1. In addition, another 88 subjects were tested or partially tested, but their data were excluded.

\section{Results}

The mean number of items recalled on the initial test for each group is presented in Table 3. The means in a given row should all be identical, but instead show considerable variability. Indeed, analysis of variance of these data produced a significant effect for retention interval $[F(5,276)=2.60]$. As expected, one presentation did not result in as many items being recalled as did three presentations $[F(1,276)=119.71]$. Although the interaction was not significant $[F(5,276)=1.77$, $\mathrm{p}=.12]$ in the context of this analysis, the probability was disturbingly low, and one must be prepared to account for some sampling bias distorting the forgetting functions.

Sample differences are a likely consequence of experiments in which retention interval is varied, due to differential mortality, although in this case there does

Table 3

Mean Number of Items Recalled on Initial Test as a

Function of Study Presentations for Groups That had had Different Retention Intervals: Experiment 2

\begin{tabular}{|c|c|c|c|c|c|}
\hline \multicolumn{6}{|c|}{ Nominal Retention Interval } \\
\hline 0 & $1 \mathrm{~h}$ & $6 \mathrm{~h}$ & 2 Days & 7 Days & 21 Day \\
\hline \multicolumn{6}{|c|}{ One Presentation } \\
\hline 6.5 & 5.5 & 6.4 & 6.2 & 6.8 & 5.5 \\
\hline \multicolumn{6}{|c|}{ Three Presentations } \\
\hline 7.8 & 9.2 & 9.9 & 9.6 & 10.5 & 9.3 \\
\hline
\end{tabular}

Note-Error mean square $=6.38$.

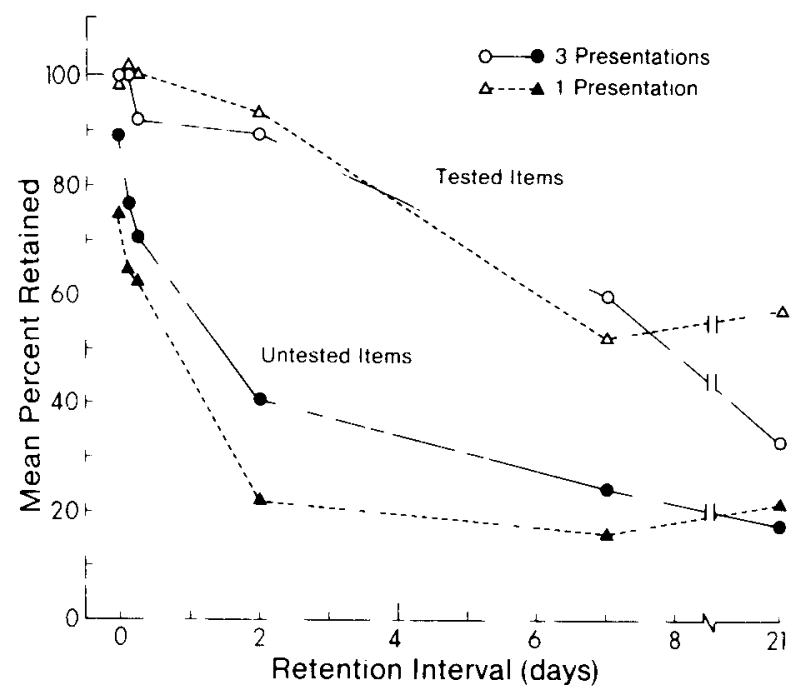

Figure 1. Mean percent retained (recall) for previously tested and previously untested items as a function of number of study presentations and retention interval: Experiment 2.

not seem to be a clear-cut relationship between level of initial recall and nominal retention interval. In any event, it may very well be an academic problem, since at least some research (Underwood, 1954) has suggested that forgetting rate is independent of learning ability.

That does not necessarily mean that forgetting is independent of initial recallability, however. For this reason, the primary measure used in analyzing the retention data was percent retained. For each subject, the number recalled on the appropriate second test was divided by the number recalled on the initial test. Although these scores do not completely compensate for any covariance between forgetting and initial recallability, they at least make direct comparisons among groups somewhat more meaningful. The mean percent retained for each group on tested and untested items is shown in Figure 1 as a function of nominal retention interval.

Whereas study presentations produced a substantial effect on the number of items retained, the percentage of items retained was virtually unaffected by this variable. The main effect of study presentations, its interaction with retention interval, and the three-way interaction all produced $F<1.00$. On the other hand, amount of study did interact with testing history $[F(1,276)=8.80]$. Despite some irregularity over retention intervals, forgetting of untested items was affected by amount of study, whereas forgetting of tested items was not. Moreover, the interaction is not due to a ceiling effect, since "negative forgetting" is clearly possible.

There was also a significant interaction between testing history and retention interval $[\mathrm{F}(5,276)=5.32]$. The interaction was produced by the fact that tested items showed little loss for up to 2 days but a decline in accessibility of untested items began almost immediately. 
Table 4

Proportion Recalled on Second Test For Items Recalled and Not Recalled on Previous Test as a Function of Number of Study Presentations and Retention Interval

\begin{tabular}{|c|c|c|c|c|c|c|}
\hline & \multicolumn{6}{|c|}{ Nominal Retention Interval } \\
\hline & 0 & $1 \mathrm{~h}$ & $6 \mathrm{~h}$ & 2 Days & 7 Days & 21 Days \\
\hline \multicolumn{7}{|c|}{ One Presentation } \\
\hline Recalled & .96 & .99 & .88 & .80 & .47 & .43 \\
\hline Not Recalled & .04 & .01 & .13 & .09 & .08 & .02 \\
\hline \multicolumn{7}{|c|}{ Three Presentations } \\
\hline Recalled & .97 & .96 & .92 & .87 & .63 & .34 \\
\hline Not Recalled & .06 & .21 & .22 & .26 & .11 & .06 \\
\hline
\end{tabular}

The result was a greater difference between tested and untested items as retention interval increased up to at least 2 days. At longer intervals, there is some convergence as forgetting approaches $100 \%{ }^{1}$

Table 4 presents the recall proportions on the second test conditionalized on initial test performance. After one presentation, the data generally follow the pattern established in Experiment 1. The retention of previously correct items decreases over time, but there is little recovery of items that had not been initially recalled, and what recovery there is, is independent of retention interval. Additional study did little to increase the recallability of previously correct items, but did result in some recovery at shorter retention intervals.

\section{Discussion}

The results of this experiment again showed that testing soon after study maintained the accessibility of items in memory. Although untested items showed gradual forgetting beginning immediately, tested items showed little loss for at least $6 \mathrm{~h}$ and forgetting was only about $10 \%$ after 2 days. Between 2 days and 7 days, the loss of tested items was greater than that for untested items, but the extent to which this is due to ceiling effects for untested items is not clear.

Nevertheless, the results again suggest that the test does not merely provide additional opportunity to study the items recalled. Additional presentations did not tend to flatten the forgetting function for untested items. In fact, additional study without test had its entire effect within the first few minutes. From that point on, the rate of forgetting was independent of study time until ceiling effects intervened. In short, testing changed the slope of the forgetting function, increased study time did not. ${ }^{2}$

At least at some points, the likelihood of recovering an initially recalled item was substantial. One thing should be pointed out about such conditional measures, however. Changes in these measures more properly reflect changes in the degree of dependence of secondtest recall on first-test recall. Even though recall was quantitatively stable for almost 2 days, the individual items became increasingly variable during this period.

\section{EXPERIMENT 3}

In the previous two experiments, substantial effort was expended to make the subjects believe that the initial memory test was the last one. The purpose of this manipulation was to prevent rehearsal during the retention interval, particularly after the subject had left the laboratory. Although some investigators (Erdelyi \& Kleinbard, 1978) have argued that such control is ecologically questionable, the study of forgetting processes seems to require such control. The question thus arose as to whether the procedures adopted in the Alberta laboratory did indeed prevent such rehearsal. If not, perhaps the tested items were maintained at the expense of untested items by being rehearsed during the retention interval at the expense of the untested items.

The primary component of the masking procedure was the Ravens Progressive Matrices test. Despite the fact that neither the content nor the procedure in this test is similar to the memory material, it might produce interference with the memories themselves or with poststudy processes contributing to later accessibility. In any event, the purpose of Experiment 3 was to evaluate the consequences of some of the masking procedures.

The experiment involved several different conditions that could be compared in different ways to yield answers to specific questions. In one set of conditions, an attempt was made to make the memory task and materials more salient by conducting the memory test in one room and the masking tests in another room. Changing rooms might result in less forgetting either because increased salience increases the amount of extraexperimental rehearsal or because the change in physical context reduces interference from the masking tasks (Strand, 1970).

In addition to these conditions, some subjects were told that the second test would be given, whereas others were not told about this test. In combination with the salience conditions, some interaction might be expected if salience affects the amount of rehearsal.

Three groups were tested without the masking tasks being given. One group was tested under what could be 
called the standard forgetting condition in that they were given the memory test and were dismissed without any mention of the second test. The second group was told to return for a retention test. The third condition represented an attempt to assess "pure" forgetting. The subjects made appointments for a single session and were given the memory task only. Some time later, they were telephoned and asked to participate in another, unrelated experiment. When they appeared for their appointment, they were unexpectedly given the second memory test. This procedure was suggested in a report by Houston (1969), except that he had the subjects recall the material over the phone. The present experiments required that the subjects recall in the same context in which study had taken place, so that it was necessary for them to return to the laboratory. However, they had no reason to try to remember the list between sessions and no reason to link the second session with the first.

\section{Method}

The word lists and the basic procedure were the same as those in Experiment 2, except that fewer retention intervals were used. The subjects received one study presentation of 24 word pairs at $5 \mathrm{sec} /$ item. For all subjects, study was followed by 2 min of work on mathematical series problems and a cuedrecall test for 12 items. The two masking tests were followed by the Ravens Progressive Matrices test, which was followed by the retention interval and the cartoon-rating test, which was followed by recall. The final cued-recall test was for all 24 items. All balancing procedures were the same as in Experiment 2.

There were 17 different conditions. One part of the experiment compared subjects given the two masking tests in the same room in which the memory test was given with subjects given the masking task in a different room. These conditions were factorially combined, with subjects being either informed or not informed about the second recall test. For each combination of these variables, retention intervals of 0,2 , and 7 days were used. Thus, there were 12 groups in this part of the experiment. Four other groups were not given the masking tasks and were tested at either 0 or 7 days. The last group was the phoned group, treated as described above, with a retention interval of 7 days.

The two rooms used for the different-rooms condition were on different floors and differed in size and in some furnishings. The memory test was given in the same room for all subjects. Transportation time was about $3 \mathrm{~min}$. The time from immediate test to the 0 -interval second test proved impossible to control and was a few minutes longer for groups that changed rooms. The subjects who had no masking task waited in a reception room for $15 \mathrm{~min}$ if they were to be tested on the 0-interval condition.

The experiment involved 408 introductory psychology students who were obtained and assigned to conditions as before. An additional 76 subjects were tested or partially tested but their data were discarded.

\section{Results and Discussion}

The mean number of items recalled on the immediate test ranged from 6.8 to 8.6. Standard errors were approximately .5 . Several different combinations of groups were compared statistically, but there were no significant differences in any of these analyses.

The percentage of items retained was computed for tested and urtested items for each subject as in Experiment 2. The means for each condition in the experiment are presented in Table 5. The results may be summarized quite succinctly. There was no evidence that any of the treatments resulted in any differences in forgetting. This conclusion is bolstered by several sets of analyses. One analysis considered only those groups given the maskıng tasks. Three effects were significant: retention interval $[F(2,276)=99.61]$, testing history $[F(1,276)=386.84]$, and their interaction $[F(2,276)=24.90]$. The interaction took the same form as in Experiment 2, with a larger difference between tested and untested items at longer retention intervals. Error mean squares for this analysis were 1,375 between subjects and 750 within subjects.

The second analysis involved all groups that did not experience the masking conditions, except the phoned group. In this analysis, retention interval $[F(1,92)=$ $135.30]$, testing history $[F(1,92)=153.51]$, and their interaction $[F(1,92)=8.41]$ were also significant, with the interaction taking the same form as above. The error mean squares were 1,113 between subjects and 442 within subjects.

The third analysis compared all of the noninformed groups (same room, different room, no masking task, and phone groups) on the 7-day test. The only significant effect was testing history $[\mathrm{F}(1,92)=302.49]$. The error mean squares were 542 between subjects and 371 within subjects.

Conditional proportions will not be presented.

Table 5

Mean Percentage of Items Retained (Recall) for Previously Tested and Previously Untested Items as a Function of Retention Interval for Varying Conditions of Masking the Retention Test

\begin{tabular}{|c|c|c|c|c|c|c|}
\hline \multirow[b]{3}{*}{ Condition } & \multicolumn{6}{|c|}{ Retention Interval } \\
\hline & \multicolumn{2}{|c|}{0} & \multicolumn{2}{|c|}{2 Days } & \multicolumn{2}{|c|}{7 Days } \\
\hline & Tested & Not Tested & Tested & Not Tested & Tested & Not Tested \\
\hline Same Room/Told & 98 & 72 & 86 & 40 & 66 & 16 \\
\hline Same Room/Not Told & 99 & 79 & 90 & 32 & 63 & 13 \\
\hline Different Room/Told & 110 & 84 & 104 & 24 & 64 & 14 \\
\hline Different Room/Not Told & 97 & 83 & 91 & 31 & 59 & 6 \\
\hline No Task/Told & 102 & 73 & & & 62 & 16 \\
\hline No Task/Not Told & 113 & 84 & & & 58 & 11 \\
\hline Phone & & & & & 62 & 19 \\
\hline
\end{tabular}


There were absolutely no differences between conditions, except for the decrease in recall of previously recalled items over time. Recall of items not previously recalled was never higher than $8 \%$ and was unrelated to experimental treatments or retention interval.

Finally, all analyses were repeated using mean number recalled and absolute loss scores (score on initial test minus score on second test). The results were statistically identical to those obtained for the percentage measure. A number of specific comparisons were made by $t$ tests. None of these comparisons was significant.

The results of the various analyses are impressively negative, and there seems to be little risk in accepting the null hypothesis. The now ubiquitous interaction of retention interval with testing history provides the only important positive finding, and this interaction apparently has little to do with differences in rehearsal during the retention interval. Indeed, it appears that little such rehearsal takes place at all. Had the phoned group done more poorly than the others, that might have indicated that the masking procedure was ineffective in preventing rehearsal, but this group actually performed better than any other group. University students apparently give rehearsal between sessions a low priority in their lives, even when they know a retention test is imminent. It also appears that the Ravens test, when given immediately after study and initial test, does not interfere with later recall.

\section{Additional Results}

The subjects in the same-room/not-told conditions of Experiment 3 were treated identically to the subjects in Experiment 2, except that they received $5 \mathrm{sec}$ of study per item rather than $3 \mathrm{sec}$ (one presentation) or $9 \mathrm{sec}$ (three presentations). This allows two comparisons across experiments that are relevant to the understanding of testing effects.

The first comparison simply underscores the fact that the interaction of testing history and retention interval is independent of amount of study. The relevant data are plotted in Figure 2. The figure simply compares the comparable conditions from the two experiments, as noted above, with the study-time differences indicated as total time per item. The data from the sameroom/not-told groups of Experiment 3 fit quite nicely into the picture provided by the other two groups.

The second comparison provides direct evidence that the effect of study and testing on rate of forgetting are not the same. This point may be made by comparing later recall for tested and untested items that are equally recallable at the 0 interval. The critical data are the untested items from the same-room/not-told group of Experiment 3 that studied each item for $5 \mathrm{sec}$ and the tested items from the single-presentation groups of Experiment 2 that studied each item for $3 \mathrm{sec}$. The former group recalled 6.6 untested items on the 0 -

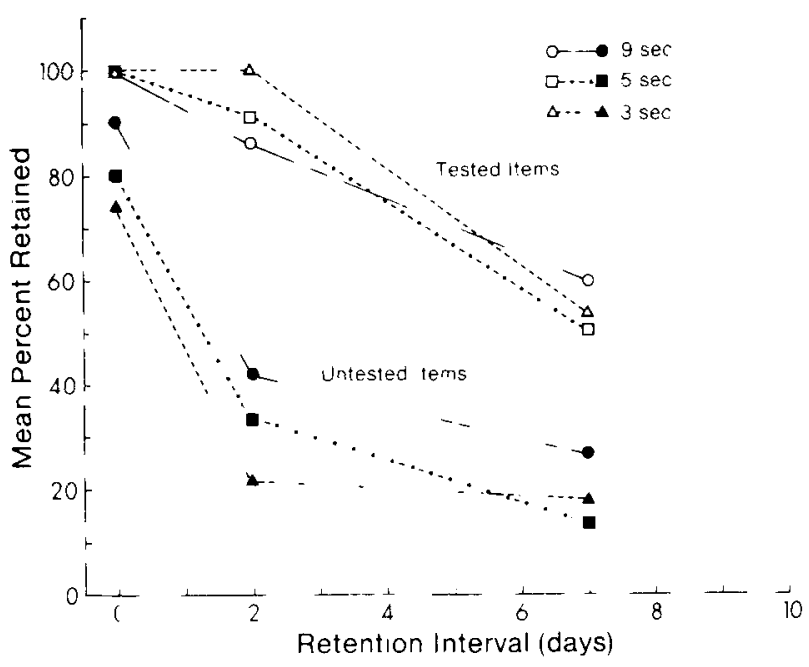

Figure 2. Mean percent retained (recall) for previously tested and previously untested items as a function of total study time and retention interval: selected groups from Experiments 2 and 3.

interval test, and the latter group recalled 6.3 tested items at the same interval. That is, the extra $2 \mathrm{sec}$ of study produced the same facilitation as the initial test. After 2 days, however, recall of tested items was 5.5 and of untested items was 2.0. After 7 days, 3.5 tested items and 1.2 untested items were recalled. Thus, despite a small advantage after about $20 \mathrm{~min}$, untested items were considerably less accessible after a few days. This result is essentially the same result reported by Thompson et al. (1978).

\section{GENERAL DISCUSSION}

Thompson and his associates (Thompson et al., 1978; Wenger et al., 1980) have provided evidence that the effects of a test on rate of forgetting are not the same as the effects of a study presentation. The results of the present series of experiments add to this evidence.

Normally, when some factor occurring prior to the retention interval has been shown to increase resistance to forgetting, the variable is interpreted as having a direct effect on the nature of the trace itself. In interpreting the results of their experiments, Wenger et al. (1980) followed this tradition. Rather than focus on the retention process, they assumed that study and test result in the encoding of different information. The interaction with retention interval is simply a result of changes in retrieval strategies over time. Differences in the encoded information are irrelevant for the retrieval process after short intervals, but become relevant as the strategy changes. The nature of the differences in the traces and/or the strategies is left unspecified. Thus, even though Wenger et al. have apparently abandoned their former opinion that study and test are essentially equivalent in their effects on the trace, they seem reluctant to consider the possibility that the processing that 
occurs on a test is totally different in kind from that occurring on a study presentation.

In many of the experiments reported by Thompson et al. (1978) and Wenger et al. (1980), the subjects in some way knew that another test was imminent during the initial test. Given such circumstances, it is reasonable to assume that the subjects would take advantage of the presence of information in active memory to further process that information. On the other hand, it is clear that most of the time spent on a test is involved in performing retrieval operations, not in reprocessing retrieved items. Moreover, in the present experiments, the subjects did not expect another test. Even in the instructed conditions of Experiment 3, they were not told about the second test until after the initial test. Given such circumstances, a substantial amount of active processing of retrieved information is unlikely.

From this perspective, the critical events occurring on a test seem to be the retrieval operations themselves. The theoretical problem then becomes one of determining how the occurrence of retrieval operations soon after study maintains the accessibility of the information over time, especially since the effects are completely item specific.

It is generally accepted principle of memory that accessibility of information is enhanced by reinstating the appropriate context, usually that present during encoding. Indeed, reinstating a physical context (Smith, 1979), an emotional mood (Bower, Monteiro, \& Gilligan, 1978), or a drug state (Eich, 1980) can enhance remembering.

One possible explanation for testing effects is that they are simply a special case of context effects. That is, the context at the time of testing includes the testing procedure and the processes involved in remembering or trying to remember. If a subject has been previousily tested, the second test essentially reinstates an important set of contextual elements for the items previously tested and successfully recalled. At short retention intervals, items are directly accessible without a great deal of this contextual support, but as direct accessibility declines over time for whatever reason, reinstating the retrieval context becomes more important.

A second possible interpretation assumes that the initial test provides the subject with the opportunity to learn successful retrieval operations for each accessible item. Irrespective of whether the process is conceptualized as a specific retrieval or search plan (Greeno, James, \& DaPolito, 1971), or as coding operations necessary to reconstruct the information (Kolers, 1973), retrieval of those items would fail only to the extent that the operations become unavailable. The interaction in this case could result from specific information in the trace becoming inaccessible at a more rapid rate than the retrieval operations.

Although there does not appear to be any way to distinguish between these two explanations at the present time, neither is denied by the facts of testing effects. The context explanation may be more attractive only in that it involves manipulable factors rather than vaguely defined internal processes. Regardless of which one of these or some other theory provides an adequate description of testing effects in this paradigm, it is probably unrealistic to expect the processes underlying these effects to apply universally in all paradigms. It is nevertheless encouraging that the results emanating from this research and those obtained by Thompson et al. (1978) and Wenger et al. (1980) are essentially identical, and appear to involve the same mechanisms despite the fact that the latter researchers used longer lists, free recall, auditory presentation, and a different design.

On the other hand, there is considerable data (e.g., Mandler \& Rabinowitz, 1981) on testing effects that points to processes that operate either in addition to or instead of the kinds of processes proposed here. The thrust of those experiments has largely been to contrast recognition and recall, an issue that the present experiments ignore. The dual-process model (familiarity and retrieval) used by Mandler and Rabinowitz to explain their results falls into the class of theories that consider processes operating on a test the same as those processes operating on a "study" exposure.

There is no issue with the above theory. The point of this research is that, when conditions are designed to minimize the further processing of specific itern information, testing effects appear to emanate from an entirely different source.

There is one other noteworthy aspect of these data. Recall of untested items after 7 days was very poor. For example, in Experiment 3, in seven groups, $41 \%$ of the 168 subjects involved failed to recall a single item. The overall mean number recalled was 1.0 item. Even after only 2 days, mean recall was only 2.1 items. These kinds of statistics underscore just how important testing is in maintaining the accessibility of information in memory. Many years ago, Underwood (1957) pointed out that the methodological proclivities of earlier years had led to an overestimation of forgetting because the phenomenon was inevitably confounded with explicit retroactive and proactive interference. It now appears that the methodological proclivities of later years have led to an underestimation of forgetting because most experiments involve multiple testing during acquisition. When experience at retrieval is not provided, successful retrieval drops off rapidly.

\section{REFERENCES}

Allen, G. A., Mahler, W. A., \& Estes, W. K. Effects of recall tests on long-term retention of paired associates. Journal of Verbal Learning and Verbal Behavior, 1969, 8, 463-470.

Bower, G. H., Monteiro, K. P., \& Gillioan, S. G. Emotional mood as a context for learning and recall. Journol of Verbal Learning and Verbol Behavior, 1978, 17, 573-585.

Eich, J. E. The cue-dependent nature of state-dependent retrieval. Memory \& Cognition, 1980, 8, 157-173.

Erdely, M. H., \& KLEINBARD, J. Has Ebbinghaus decayed with 
time? The growth of recall (hypermnesia) over days. Journal of Experimental Psychology: Human Learning and Memory, 1978, 4, 275-289.

Greeno, J. G., James, C. G., \& DaPolito, F. J. A cognitive interpretation of negative transfer and forgetting of paired associates. Journal of Verbal Learning and Verbal Behavior, 1971, 10, 331-345.

Houston, J. P. Proactive inhibition and undetected retention interval rehearsal. Journal of Experimental Psychology, 1969, 82, 511-514.

KoLers, P. Remembering operations. Memory \& Cognition, 1973, 1, 347-355.

LAPorte, A., \& Voss, J. F. Retention of prose materials as a function of post acquisition testing. Journal of Educational Psychology, 1975, 67, 259-266.

LofTUS, G. R. On interpreting interactions. Memory \& Cognition, 1978, 6, 312-319.

Mandler, G., \& Rabinowitz, J. C. Appearance and reality: Does a recognition test really improve subsequent recall and recognition? Journal of Experimental Psychology: Human Learning and Memory, 1981, 7, 79-90.

Martin, E., Fleming, F. G., \& Nally, P. D. Effect of temporal locus of a recitation attempt on learning and retention. Memory \& Cognition, 1978, 6, 274-282.

Runquist, W. N. Conditional recall and bias in memory experiments. American Journal of Psychology, 1973, 86, 351-360.

Stamecka, N., \& McElree, B. Normal forgetting of verbal lists as a function of their degree of learning. Journal of Experimental Psychology: Learning, Memory, and Cognition, 1983, 9, 384-397.

Smith, S. T. Remembering in and out of context. Journal of Experimental Psychology: Human Learning and Memory, 1979, 5, 460-471.

Strand, B. Z. Change of context and retroactive inhibition. Journal of Verbal Learning and Verbal Behavior, 1970, 9, 202-206.

Thompson, C. P., Wenoer, S. K., \& Bartlina, C. A. How recall facilitates subsequent recall; A reappraisal. Journal of Experimental Psychology: Human Learning and Memory, 1978, 4, 210-221.

Tulving, E. Intratrial and intertrial retention: Notes toward a theory of free recall verbal learning. Psychological Review, 1964, 71, 219-237.
Tulving, E., \& Thomson, D. M. Encoding specificity and retrieval processes episodic memory. Psychological Review, 1973, 80, 352-373.

UNDERWOOD, B. J. Speed of learning and amount retained: A study in methodology. Psychological Bulletin, 1954, 51, 276-282.

UNDE RWOOD, B. J. Interference and forgetting. Psychological Review, 1957, 64, 49-60.

Wenger, S. K., Thompson, C. P., \& Baftling, C. A. Recall facilitates subsequent recognition. Journal of Experimental Psychology: Human Learning and Memory, 1980, 6, 135-144.

\section{NOTES}

1. In dealing with interactions over retention intervals, the so-called "scaling" problem is critical. Indeed, if one simply considers the number of items recalled on the second test or the number of items lost (initial test recall-second test recall), one might expect differences. Purely additive variables using these measures may interact when converted to percentage measures. Thus, the conclusion one reaches about such interactions depends upon the scale used (Loftus, 1978).

For whatever the reason, neither of the interactions reported here depends upon the scale, although the theoretically important interaction of testing history with study trials did not quite achieve significance with either items recalled or absolute loss ( $p=.06$ in both cases).

In general, however, the problem is not trivial. In many cases, there is no rational reason for choice of a particular measure. In this case, when comparing forgetting functions with different initial points, the percentage measure seems more appropriate because it takes this difference into account. Not all researchers agree, however (see, e.g., Slamecka \& McElree, 1983).

2. With the absolute-loss measures, the untested items for one- and three-presentation groups had different slopes. The curves were identical up to $6 \mathrm{~h}$ and then diverged, but the flattening of the retention function between 0 and 2 days, which was characteristic for tested items, was not apparent.

(Manuscript received August 2, 1982; revision accepted for publication May 27, 1983.) 